\title{
Cytoreductive Surgery and Hyperthermic Intraperitoneal Chemotherapy for Peritoneal Carcinomatosis
}

\author{
Hadi M. Khan and Hamza Hanif \\ Department of Surgery, Shifa International Hospital, Islamabad, Pakistan
}

\begin{abstract}
Peritoneal carcinomatosis (PC) is a common presentation of several gastrointestinal and gynecological malignancies. Cytoreductive surgery and hyperthermic intraperitoneal chemotherapy (CRS + HIPEC) is a treatment regimen recently introduced in Pakistan for PC. The goal of cytoreductive surgery is complete removal of macroscopic disease. HIPEC is administered following surgery, with the aim of eliminating disease at a microscopic level. In this study, 11 patients, who underwent CRS+HIPEC at the Shifa International Hospital, Islamabad, Pakistan, were selected. Disease severity was classified using PCl score. There were $54.5 \%$ women and $45.5 \%$ men with mean age of $48.5 \pm 12.5$ years. The mean PCl score was $20.3 \pm 3.4$. The mean time from diagnosis was $12.7 \pm 11.6$ months. A complete tumor resection (CC-0) was achieved in 10 (90.9\%) patients, while the rest were CC-1. The duration of HIPEC circulation was 90 minutes in every patient. Postoperative morbidity was observed in 2 (18.2\%) patients. No 30-day perioperative mortality was seen. It was concluded that with effective patient selection, surgical skills and center experience, CRS+HIPEC can have low perioperative morbidity and mortality, and complete cytoreduction leads to prolonged overall survival.
\end{abstract}

Key Words: Peritoneal carcinomatosis, Cytoreductive surgery, Hyperthermic intraperitoneal chemotherapy.

How to cite this article: Khan HM, Hanif H. Cytoreductive Surgery and Hyperthermic Intraperitoneal Chemotherapy for Peritoneal Carcinomatosis. J Coll Physicians Surg Pak 2022; 32(02):259-261.

Various gynecological and gastrointestinal malignancies present with peritoneal carcinomatosis (PC). PC is a Stage 4 disease, for which only palliative treatment was given traditionally. Once diagnosed, survival is generally less than six months. ${ }^{1}$ In 1980s, the idea of treating PC as a locally advanced disease and to manage it with aggressive intervention was suggested. Since then, the use of cytoreductive surgery and hyperthermic intraperitoneal chemotherapy (CRS+HIPEC) has gained popularity as a treatment regimen with curative intent and prolonged survival. The goal of cytoreductive surgery is complete removal of macroscopic disease. HIPEC is administered following the surgery, with the aim of eliminating disease at a microscopic level. Therapeutic levels of chemotherapy can be achieved with much lower doses compared to the intravenous chemotherapeutic regimen, avoiding major systemic side effects. Hyperthermia $\left(40-42^{\circ} \mathrm{C}\right)$ further enhances effectiveness of the chemotherapy by direct cytotoxicity, improving tissue permeability, and increasing peritoneal blood flow.

Correspondence to: Dr. Hamza Hanif, Department of Surgery, Shifa International Hospital, Pitras Bukhari, Islamabad, Pakistan

E-mail: hamza.hanif745@gmail.com

Received: July 13, 2021; Revised: November 05, 2021;

Accepted: November 15, 2021

DOI: https://doi.org/10.29271/jcpsp.2022.02.259
Complete cytoreduction in patients with extensive peritoneal disease requires major surgery with high morbidity and mortality. Yet, recent literature shows that curative approach to PC using CRS and HIPEC may prolong survival. In colorectal cancer, the median overall survival (OS) can be significantly increased with CRS + HIPEC to up to 21-63 months with 5-year OS of up to more than $40 \% .{ }^{2}$ For appendiceal neoplasms, CRS + HIPEC can improve median survival up to 100 months, with a 5year OS of up to $71 \%{ }^{3}$ Similarly, the OS in patients with ovarian cancer has also been significantly improved with CRS + HIPEC. ${ }^{4}$ Despite these results, very few centers in Pakistan perform this procedure. It is being performed in Shifa International Hospital (SIH) since 2019.

The aim of this study was to analyse the safety, effectiveness, peri and postoperative outcomes of CRS+HIPEC at a tertiary care centre in Pakistan.

This study was approved by Institutional Review Board of SIH, Islamabad, Pakistan. All data was analysed using SPSS23.0.

This prospective observational study included eleven patients with peritoneal carcinomatosis originating from the appendix, ovaries, and colon. Candidate patients were selected based on multiple factors including age, cardiopulmonary function, performance status, and peritoneal cancer index ( $\mathrm{PCl}$ score). Preoperative staging was done using $\mathrm{CT}$ scans and tumor markers (CEA, CA 125). Candidate selection and indications for treatment were established in multi-disciplinary tumor board. Written informed consents were obtained from all patients. The 
extent of peritoneal disease was evaluated using the $\mathrm{PCI}$ score, an index used to assess the extent of peritoneal cancer based on 13 well-defined abdominal regions (Figure 1 ). This score was calculated directly after explorative laparotomy and before CRS procedures, and was simultaneously recorded and calculated byanassistant.

Regions
0 Central
1 Right Upper
2 Epigastrium
3 Left Upper
4 Left Flank
5 Left Lower
6 Pelvis
7 Right Lower
8 Right Flank

Figure 1: Peritoneal cancerindex.

Table I: Completeness of cytoreduction score.

\begin{tabular}{|l|l|}
\hline Score & Definition \\
\hline CC-0 & No peritoneal nodule was seen \\
\hline CC-1 & $\begin{array}{l}\text { Tumor nodules persisting after cytoreduction are less } \\
\text { than } 2.5 \mathrm{~mm} \text { in diameter }\end{array}$ \\
\hline CC-2 & $\begin{array}{l}\text { Tumor nodules persisting after cytoreduction are } \\
\text { between } 2.5 \mathrm{~mm} \text { and } 2.5 \mathrm{~cm} \text { in diameter }\end{array}$ \\
\hline CC-3 & $\begin{array}{l}\text { Tumor nodules persisting after cytoreduction are greater } \\
\text { than } 2.5 \mathrm{~cm} \text { in diameter }\end{array}$ \\
\hline
\end{tabular}

Aggressive CRS was carried out with the primary objective of achieving complete cytoreduction, using procedures including but not limited to exploratory laparotomy, abdominal wall resection, bowel resection, lymphadenectomy, enterolysis, ureterolysis, peritonectomy, and omentectomy. Completeness of cytoreduction score (CC score) was used to determine success of CRS (Table I). Following the cytoreduction, HIPEC was administered. The chemotherapeutic regimen varied for different tumor entities. Pre-heated $\left(40-42^{\circ} \mathrm{C}\right)$ HIPEC was then delivered for 90 minutes in a closed abdomen in each case, using Mytomycin at $25 \mathrm{mg} / \mathrm{m}^{2}$ or Cisplatin at $30 \mathrm{mg} / \mathrm{m}^{2}$.

There were $54.5 \%$ women and $45.5 \%$ men with mean age of $48.5 \pm 12.5$ years. The mean $\mathrm{PCI}$ score was $20.3 \pm 3.4$. The mean time from diagnosis was $12.7 \pm 11.6$ months. ECOG performance score was 0 in seven (63.6\%) patients, 1 in three $(27.3 \%)$ patients, and 2 in one (9.1\%) patient. Seven patients $(63.6 \%)$ underwent neoadjuvant chemotherapy. The primary diagnosis was mucinous neoplasm of the appendix in seven $(63.6 \%)$ patients, high-grade ovarian carcinoma in one $(9 \%)$ patient, and colorectal cancer in three $(27.3 \%)$ patients. A complete tumor resection (CC-0) was achieved in 10 (90.9\%) patients, while the rest were $\mathrm{CC}-1$. The duration of HIPEC circulation was 90 minutes in every patient. The mean temperature of the perfu- sion was $41 \pm 0.5^{\circ} \mathrm{C}$. The mean operative time (surgery and HIPEC) was $9.8 \pm 0.8$ hours. All patients were shifted to the SICU as per protocol. The mean length of hospital stay was $8.2 \pm 0.8$ daysand ICU stay was $3.2 \pm 0.8$ days.

Postoperative morbidity was observed in 2 (18.2\%) patients. One patient with appendiceal neoplasm developed abdominal pain and significant drop in hemoglobin on postoperative day one; and was shifted to the operating room. The bleeding was controlled during re-exploration, after which the patient had an uncomplicated hospital stay. Another patient with appendiceal neoplasm developed reversible cardiomyopathy (ejection fraction of $25 \%$ ) postoperatively. Her ICU stay was prolonged by two days. Echocardiography was repeated on her 2-month followup, which showed an ejection fraction of $50 \%$. No 30-day peri-operative mortality was seen.

The factors associated with perioperative morbidity in these patients include disease dissemination, extent of cytoreduction, the number of removed organs, age, and operative time. ${ }^{5}$ Another major risk factor for morbidity is HIPEC-related toxicity. For this reason, a thorough preoperative evaluation including kidney function, and close early postoperative monitoring is necessary. Furthermore, surgeon and centre experience are also significant factors affecting morbidity and mortality. The learning curve is not only for the surgeons but also the institution, since this type of treatment requires decision making on a multidisciplinary level.

Among these factors, completeness of cytoreduction (CC score), $\mathrm{PCl}$ score, and histological grade have been shown to be the most significant prognostic factors for perioperative and long-term survival. ${ }^{5}$ The $\mathrm{PCl}$ score is commonly used tool to quantify the extent of tumor growth based on the region and size of metastatic nodules. The CC-score is another important quantitative prognostic factor. A complete cytoreduction indicates that the surgeon successfully cleared all visible sites of disease (CC-0) or left behind only a few minute nodules (CC-1 = $<2.5 \mathrm{~cm}$ ) which are expected to be eradicated by HIPEC. Various studies including an analysis of a prospective database at a national peritoneal tumor center demonstrated significantassociation between a low CC score with higher OS. ${ }^{6}$

Patient selection for curative approach is extremely important and multifactorial. These factors are related to the patient and the tumor in combination. The tumor-related factors include tumor origin, histological grade, presence of metastatic disease, lymph node involvement, response to previous chemotherapies, and $\mathrm{PCl}$ score. Patient related factors include patient performance status, co-morbidities, and multidisciplinary tumor board decision. Imaging studies including CT scans are also mandatory for patientselection.

CRS + HIPEC is offered at a limited number of healthcare facilities in Pakistan, due to lack of expertise and economic burden. However, it is a highly effective treatment modality, which is being used worldwide for PC originating from gastrointestinal or gynecologicalcancers. With effective patient selection, the peri- 
operative morbidity and mortality is low, and complete cytoreduction leads to a significantly prolonged overall survival.

\section{PATIENTS' CONSENT:}

Written informed consents were obtained from all patients.

\section{CONFLICT OF INTEREST:}

The authors declared no conflict of interest.

\section{AUTHORS' CONTRIBUTION:}

HMK: Operated in all cases, drafted the design of the study, conducted literature review and drafted the manuscript.

$\mathrm{HH}$ : Collected data from medical records, conducted statistical analysis and drafted the manuscript.

\section{REFERENCES}

1. Spiliotis JD, Halkia E, Boumis VA, Vassiliadou DT, Pagoulatou A, Efstathiou E. Cytoreductive surgery and HIPEC for peritoneal carcinomatosis in the elderly. Int J Surg Oncol 2014; 2014. doi: 10.1155/2014/987475.

2. Huang CQ, Min Y, Wang SY, Yang XJ, Liu Y, Xiong B, et al. Cytoreductive surgery plus hyperthermic intraperitoneal chemotherapy improves survival for peritoneal carcinomatosis from colorectal cancer: A systematic review and meta-analysis of current evidence. Oncotarget 2017; 8(33):55657. doi: 10.18632/oncotarget.17497.

3. Narasimhan V, Wilson K, Britto M, Warrier S, Lynch AC, Michael $M$, et al. Outcomes following cytoreduction and HIPEC for pseudomyxoma peritonei: 10-year experience. J Gastrointes Sur 2020; 24(4):899-906. doi: 10.1007/s11605019-04239-4.

4. Zhang G, Zhu Y, Liu C, Chao G, Cui R, Zhang Z. The prognosis impact of hyperthermic intraperitoneal chemotherapy (HIPEC) plus cytoreductive surgery (CRS) in advanced ovarian cancer: The meta-analysis. J Ovarian Res 2019; 12(1):1-9. doi: 10.1186/s13048-019-0509-1.

5. Kusamura S, Younan R, Baratti D, Costanzo P, Favaro M, Gavazzi C, et al. Cytoreductive surgery followed by intraperitoneal hyperthermic perfusion: Analysis of morbidity and mortality in 209 peritoneal surface malignancies treated with closed abdomen technique. Cancer 2006; 106(5):1144-53. doi: 10.1002/cncr.21708.

6. Aziz O, Jaradat I, Chakrabarty B, Selvasekar CR, Fulford PE, Saunders MP, et al. Predicting survival after cytoreductive surgery and hyperthermic intraperitoneal chemotherapy for appendix adenocarcinoma. Dis Colon Rectum 2018; 61(7):795-802. doi: 10.1097/DCR.0000000000001076. 